\title{
Superconducting properties of the $\mathrm{Bi}-\mathrm{Ca}-\mathrm{Sr}-\mathrm{Cu}-\mathrm{O}$ system
}

\author{
S M M R NAQVI, MUSHEER H KHAN, A A QIDWAI, FIROZ \\ AHMAD, S M ZIA-UL-HAQ and RASHID-BIN SAIF \\ Department of Physics, University of Karachi, Karachi 75270, Pakistan
}

\begin{abstract}
The electrical properties of unquenched and rapidly quenched bulk samples of granular $\mathrm{Bi}_{2}-\mathrm{Ca}_{1}-\mathrm{Sr}_{2}-\mathrm{Cu}_{2}-\mathrm{O}_{x}$ system have been studied. Electrical resistivity measurements show superconductivity transition temperature $(R=0)$ at $72 \mathrm{~K}, 80 \mathrm{~K}$ and $90 \cdot 5 \mathrm{~K}$. X-ray crystallographic studies show that most samples were of single-phase.
\end{abstract}

Keywords. Electrical resistivity; superconducting transition; X-ray crystallography.

\section{Introduction}

High temperature superconductivity in $\mathrm{Bi}-\mathrm{Ca}-\mathrm{Sr}-\mathrm{Cu}-\mathrm{O}$ has attracted considerable attention (Maeda et al 1988; Rao 1988; Hazen et al 1988; Tarascon et al 1988; Rao et al 1988). X-ray diffraction studies have also been carried out by many research groups (Bordet et al 1988; Takayama et al 1988; Syono et al 1988) to identify and characterize the superconducting phase. The $80 \mathrm{~K}$ superconductivity phase has been reported to have a composition of $\mathrm{Bi}_{2} \mathrm{Ca}_{1} \mathrm{Sr}_{2} \mathrm{Cu}_{2} \mathrm{O}_{x}$ while the $110 \mathrm{~K}$ phase has a possible composition of $\mathrm{Bi}_{2} \mathrm{Ca}_{2} \mathrm{Sr}_{2} \mathrm{Cu}_{3} \mathrm{O}_{y}$ (Zandenbergen et al 1988). It is clear that this class of materials encompasses several distinct superconducting phases and that the physical properties measured greatly depend not only on the elemental composition but on the preparation details. The present paper reports the preparation of two batches of superconducting samples (quenched and unquenched) and the measurement of their electrical resistivity and $\mathrm{X}$-ray diffraction studies to understand the basic feature of the $\mathrm{Bi}-\mathrm{Ca}-\mathrm{Sr}-\mathrm{Cu}-\mathrm{O}$ system.

\section{Experiment}

Bulk sample of 2:1:2:2 composition was prepared by the solid-state reaction of $\mathrm{Bi}_{2} \mathrm{O}_{3}, \mathrm{CuO}, \mathrm{SrCO}_{3}$ and $\mathrm{CaCO}_{3}$ powders. The method of synthesis consisted of reacting together $\mathrm{Bi}_{2} \mathrm{O}_{3}$ and a base matrix of $\mathrm{CaSr}_{2} \mathrm{Cu}_{2} \mathrm{O}_{5}$. The matrix was prepared by thoroughly mixing appropriate quantities of $\mathrm{CaCO}_{3}, \mathrm{SrCO}_{3}$ and $\mathrm{CuO}$ by heating in an alumina boat at $800-900^{\circ} \mathrm{C}$ with intermittent grindings. The reacted powder was cooled, ground and pelletized and was heated for a couple of hours. One batch of pellets was slowly removed from the furnace and cooled to room temperature (unquenched), while another batch was removed from the furnace and quenched by immersing in liquid nitrogen. The pellets were cut in parallelopiped shape to measure the electrical resistivity. Contacts were made using silver paste in the sample surface. Resistivity was measured by the conventional four-probe method. An XY plotter was used to measure voltage and temperature. The temperature was measured using a copper-constantan thermocouple. 

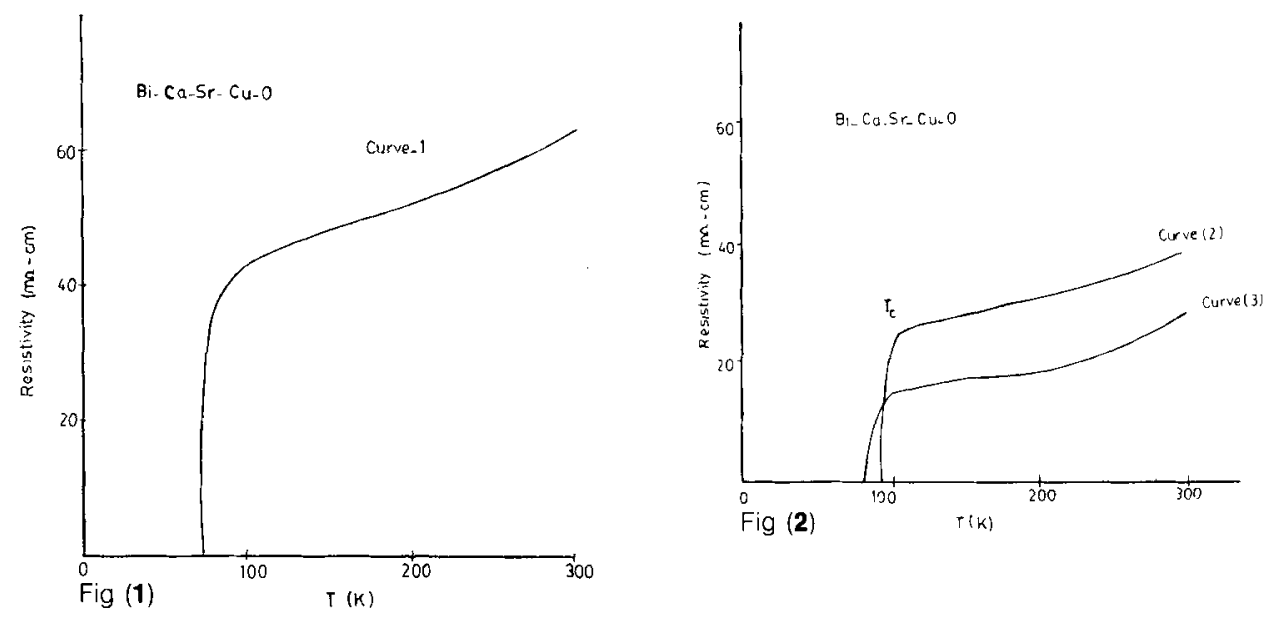

Figures 1 and 2. Resistivity vs temperature of $\mathrm{Bi}_{2} \mathrm{Ca}_{1} \mathrm{Sr}_{2} \mathrm{Cu}_{2} \mathrm{O}_{x}$ at current density of $15 \mathrm{~mA} / \mathrm{mm}^{2}$. Curve 1. Unquenched sample. Curve 2. Quenched sample. Curve 3. For quenched sample, taken 15 days after that in curve 2 .

The experimental details of the measuring technique were reported earlier (Naqvi et al 1989).

\section{Results and discussion}

$R-T$ curves for typical unquenched and rapidly quenched specimens are shown in figures 1 and 2. The resistivity of the unquenched sample from batch (I) shows metallic temperature behaviour down to the superconducting onset at $90 \mathrm{~K}$, while zero resistance $T_{c}$ is observed at $72 \mathrm{~K}$. From batch (I) most of the unquenched samples showed a zero resistance around $72-75 \mathrm{~K}$. Figure 1 shows the typical behaviour of one of the representative samples belonging to batch (I). The measurements have been done at a current density of $15 \mathrm{~mA} / \mathrm{mm}^{2}$. The $T_{c}$ of the sample did not degrade with increase of current density. This indicates that the sample can endure current density variations. The transition width in the sample is around $18 \mathrm{~K}$.

The resistivity of the rapid quenched sample from batch (II) in figure 2 showed improved metallic behaviour above the superconducting onset around $105 \mathrm{~K}$, while zero resistance was found at $90.5 \mathrm{~K}$. None of the samples showed any evidence of a second onset at $105-110 \mathrm{~K}$ as reported in literature (Shi et al 1988) for samples deviating from the $2: 1: 2: 2$ composition. Some of our rapidly-quenched samples were found to attain the behaviour of unquenched material over a few days when exposed to the atmosphere at ambient temperatures. The resistivity curve shows $T_{c}$ onset around $94 \mathrm{~K}$ and zero resistance at $80 \mathrm{~K}$. The changes in $T_{c}$ and electrical resistivity are attributed to the uptake of atmospheric oxygen as pointed out earlier (Tallon et al 1988; King et al 1989).

The specimen samples of batches (I) and (II) were examined by X-ray diffractometer after crushing. The crystal structure was found to be orthorhombic (Bordet et al 1988). X-ray crystallographic studies showed that most of the samples were of single phase. 


\section{Conclusions}

By using a matrix method it is possible to prepare pure superconductivity phase in the $\mathrm{Bi}-\mathrm{Ca}-\mathrm{Sr}-\mathrm{Cu}-\mathrm{O}$ system starting from stoichiometric ratios (2122) of the constituent oxides. Also superconducting phase (2122) can be prepared from quenching and slow cooling (unquenched).

\section{Acknowledgements}

We are grateful to Professor S A Hussain of University of Karachi, and Dr Y Khan of the Institute fur Werkstoffe der Electrotechnik, Ruhr Universitat, Bochum, for experimental facilities. Financial assistance from the National Scientific Research and Development Board (NSRDB) and the University of Karachi for this work is greatly acknowledged. One of us (MHK) is grateful to ICTP, Trieste, Italy for facilities in the Adriatico Laboratory.

\section{References}

Bordet P et al 1988 Interlaken Conf. Physica $\mathbf{C 1 5 3}$

Hazen R M et al 1988 Phys. Rev. Lett. 601174

King P J, Misra D S and Roys W B 1989 Supercond. Sci. Technol. 1227

Maeda H, Tanaka Y, Fukutomi M and Asano T 1988 Jpn. J. Appl. Phys. Lett. 27209

Naqui S M M R, Alam J, Ahmad F and Khan M H 1989 Int. J. Mod. Phys. 3 441, 661

Rao C N R 1988 Chemistry of oxide superconductors (Oxford: Blackwell)

Rao C N R, Mohan Ram R A, Ganapathi L and Vijayaraghavan R 1988 Pramana-J. Phys. 30 L495

Shi D, Blank M, Patel M, Hinks D G and Mitchell A W 1988 Physica C156 822

Syono Y et al 1988 Jpn. J. Appl. Phys. 27 L569

Tallon J L, Buckley R G, Gilberd P W, Presland M R, Brown I W M, Bowden M E, Christion L A and Goguel R 1988 Nature (London) 333153

Takayama-Muromachi E et al 1988 (to appear in Jpn. J. Appl. Phys.)

Tarascon J M, Le Page Y, Barboux P, Bagley B G, Greene L H, Mckinnon W R, Hull G W, Giroundi $M$ and Hwang D M 1988 Phys. Rev. B37 9382

Zandenbergen H W, Huang Y K, Menken M J V, Li J N, Kadowaki K, Menovsky A A, Van Tendelloo G and Amelinck X S 1988 Solid State Commun. 66297 